PHYSICAL REVIEW B 80, 180501(R) (2009)

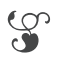

\title{
Spontaneous fluxoid formation in superconducting loops
}

\author{
R. Monaco* \\ Istituto di Cibernetica del CNR, 80078 Pozzuoli, Italy \\ and Unità INFM Dipartimento di Fisica, Università di Salerno, 84081 Baronissi, Italy \\ J. Mygind ${ }^{\dagger}$ \\ DTU Physics, B309, Technical University of Denmark, DK-2800 Lyngby, Denmark \\ R. J. Rivers ${ }^{\ddagger}$ \\ Blackett Laboratory, Imperial College London, London SW7 2AZ, United Kingdom
}

\author{
V. P. Koshelets ${ }^{\S}$ \\ Kotel'nikov Institute of Radio Engineering and Electronics, Russian Academy of Science, Mokhovaya 11, Bldg 7, 125009 Moscow, Russia \\ (Received 24 July 2009; published 2 November 2009)
}

\begin{abstract}
We report on the experimental verification of the Zurek-Kibble scenario in an isolated superconducting ring over a wide parameter range. The probability of creating a single flux quantum spontaneously during the fast normal-superconducting phase transition of a wide $\mathrm{Nb}$ loop clearly follows a scaling relation on the quenching time $\tau_{Q}$, as one would expect if the transition took place as fast as causality permits. However, the observed Zurek-Kibble scaling exponent $\sigma=0.62 \pm 0.15$ is two times larger than anticipated for large loops. Assuming Gaussian winding number densities we show that this doubling is well founded for small annuli.
\end{abstract}

DOI: 10.1103/PhysRevB.80.180501

PACS number(s): 74.50.+r, 67.25.dk, 05.70.Fh, 11.27.+d

The Zurek-Kibble (ZK) scenario ${ }^{1-3}$ proposes that continuous phase transitions take effect as fast as possible i.e., the domain structure after the quenching of the system initially reflects the causal horizons. This proposal can be tested directly for transitions whose domain boundaries carry visible topological charge. In this Rapid Communication we shall show that, with qualifications, this scenario is strongly corroborated by the behavior of superconducting loops, for which the topological defect is a fluxoid i.e., a supercurrent vortex carrying one magnetic flux quantum $\Phi_{0}=h /(2 e)$ $\simeq 2.08 \times 10^{-15} \mathrm{~Wb}$.

The basic scenario is very simple. Consider a planar low- $T_{c}$ superconductor in which a hole has been made of circumference $C$. In the Meissner state the order parameter for the superconductor is a complex field $\psi$ with phase $\phi$, $\psi=\rho e^{i \phi}$, where $|\rho|^{2}$ measures the density of Cooper pairs. On quenching the system from the normal to superconducting phase, causality prevents the system from adopting a uniform phase. If, on completion of the quench, we follow the periodic phase $\phi(x)(\bmod 2 \pi)$ along the boundary of the hole (coordinate $x$ ), we can define a winding number density: $n(x)=d \phi(x) / d x /(2 \pi)$. The total normalized magnetic flux through the hole is, in units of $\Phi_{0}$, the winding number,

$$
n=\int_{0}^{C} n(x) d x=\frac{\Delta \phi}{2 \pi},
$$

where $\Delta \phi$ is the change in $\phi$. In the absence of an external magnetic field, on average $\langle n\rangle=0$, but it will have nonzero variance $(\Delta n)^{2}=\left\langle n^{2}\right\rangle$, which is what can be measured in terms of the probabilities $f_{ \pm m}$ to trap \pm m flux quanta: $\left\langle n^{2}\right\rangle$ $=\sum_{m=-\infty}^{\infty} m^{2} f_{m}$. According to Ref. 2, on completion of a thermal quench having a given inverse quench rate $\tau_{Q}=-T_{c} /(d T / d t)_{T=T_{c}}$, the phase $\phi$ is correlated over distances
$2 \pi \bar{\xi}$, where $\bar{\xi}$ was predicted to scale ${ }^{2}$ with the quench time $\tau_{Q}$

$$
\bar{\xi} \approx \xi_{0}\left(\frac{\tau_{Q}}{\tau_{0}}\right)^{\sigma}
$$

$\bar{\xi}$, also called the ZK causal length, is defined in terms of the cold correlation length $\xi_{0}$ and the Ginzburg-Landau relaxation time $\tau_{0}$ of the long wavelength modes. The ZK scaling exponent $\sigma$ is determined by the static critical exponents of the system and, in the mean-field approximation, $\sigma=1 / 4 .^{1}$ If we make the further assumption that there is a random walk in phase on a scale $2 \pi \bar{\xi}$ then, for a hole of radius $r$, circumference $C \gg 2 \pi \bar{\xi}$,

$$
\left\langle n^{2}\right\rangle \approx \frac{C}{2 \pi \bar{\xi}}=\frac{r}{\xi_{0}}\left(\frac{\tau_{Q}}{\tau_{0}}\right)^{-\sigma} .
$$

For small rings with $C<2 \pi \bar{\xi}$ the likelihood of seeing two or more units of flux is small and $\left\langle n^{2}\right\rangle \approx f_{+1}+f_{-1}=f_{1}$, the probability of single fluxoid trapping. It is plausible to extrapolate Eq. (3) to

$$
f_{1} \approx\left\langle n^{2}\right\rangle \approx \frac{r}{\xi_{0}}\left(\frac{\tau_{Q}}{\tau_{0}}\right)^{-\sigma},
$$

showing scaling behavior of $f_{1}$ with the same exponent. We note that the $\mathrm{ZK}$ argument makes no assumptions about the rest of the superconductor, equally valid for the phase change along the inner circumference of an annulus as it is for the phase change around a single hole in a superconducting sheet. In 2003 the first experiment with superconducting loops ${ }^{4}$ was performed to test Eq. (3). The experiment consisted of taking an isolated array of thin-film wide rings and 
making it undergo a forced phase transition by heating it above its superconducting critical temperature and letting it to cool passively back toward the $L H$ e temperature. Once the thermal cycle is over, the rings are inspected by a scanning superconducting quantum interference device and the number and polarity of any trapped fluxoids determined. These rings, of amorphous $\mathrm{Mo}_{3} \mathrm{Si}$ thin films, had thickness almost one order of magnitude smaller than the low temperature London penetration depth. Although this provides favorable conditions for thermally activated phenomena it drastically increases the likelihood that nucleated vortices escape through the ring walls during the fast quench. In fact, the experimental outcome was totally at variance with the scaling above. However, the prediction [Eq. (3)] presupposes that we can ignore the contribution to the flux from the freezing in of thermal fluctuations of the magnetic field ${ }^{5}$ and the results of Ref. 4 could be explained in terms of the freezing of thermally activated fluxoids in a similar spirit to Ref. 5.

In this Rapid Communication we shall present results from an experiment with high-quality $\mathrm{Nb}$ film rings with $r$ $=30 \mu \mathrm{m}$, two times thicker than their low temperature London penetration length $\lambda_{L, N b}$; the film thickness and composition were chosen to reduce the thermal activation of fluxoids and, at the same time, 'the washing out' of fluxoids generated by the conventional causality mechanism. In our case the contribution $\Delta f_{1}$ to the probability of finding a unit of flux from thermal magnetic field fluctuations is approximately ${ }^{5}$

$$
\Delta f_{1} \lesssim\left(k_{B} T_{c}\right) r \mu_{0} / \Phi_{0}^{2} \approx 6 \times 10^{-4},
$$

and can be safely ignored. We therefore look for scaling behavior in $\tau_{Q}$.

Here a different way of counting both the number and the polarity of generated defects has been adopted. It is based on the detection of the persistent currents $\mathbf{J}_{\mathbf{s}}$ circulating around a hole in a superconducting film, when one or more fluxoids are trapped inside the hole. The circulating currents screening the bulk of the superconductor from the trapped flux induce a magnetic field $\mathbf{H}$ in the volume around the ring, such that $\mathbf{J}_{\mathbf{s}}=\boldsymbol{\nabla} \times \mathbf{H}$. By placing a Josephson tunnel junction (JTJ) along the perimeter of the hole in the area where this field passes, any trapped fluxoid will result in a modulation of the JTJ critical current, similar to the effect of an external field applied perpendicular to the ring. Indeed, this method is strongly inspired by the results found investigating the effects of a transverse field on Josephson junctions of various geometries. ${ }^{6}$ The geometry of our experiment is sketched in Fig. 1; the black wide ring is a 200-nm-thick Nb film, which also acts as the common base electrode for two JTJs whose top electrodes are depicted in gray. The JTJs have the shape of gapped annuli and the bias current is supplied in their middle point; this geometrical configuration is characterized by a peculiar magnetic diffraction pattern: for small magnetic fields the critical current increases both for positive and negative field values. ${ }^{15}$ The original purpose of having two counter electrodes on the base ring was that any screening current circulating on the outer ring circumference will preferentially affect the outermost JTJ, and vice versa for the screening current on the inside of the ring. Since the persis-

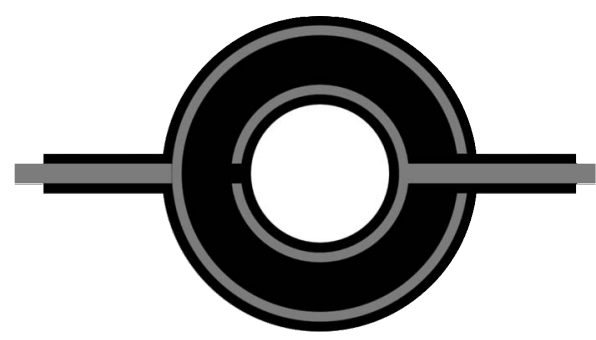

FIG. 1. Sketch of a superconducting loop (black) used as a base electrode for two gapped $\mathrm{Nb} / \mathrm{AlOx} / \mathrm{Nb}$ annular Josephson tunnel junctions (whose top electrodes are in gray). The ring inner and outer radii are $r=30$ and $R=50 \mu \mathrm{m}$, respectively, while the top electrodes width is $5 \mu \mathrm{m}$.

tent currents due to trapped flux mainly flow in the inner ring circumference, ${ }^{7}$ in this experiment we only used the innermost JTJ. The layout shown in Fig. 1, with a few key differences, bears remarkable topological similarity to the one used in a series of experiments by us to demonstrate the $\mathrm{ZK}$ scaling behavior of Eq. (3) in annular JTJs. ${ }^{8-12}$ The most obvious difference in the design is the inclusion of the two junction counter electrodes on top of the ring-shaped base electrode. The second change is the removal of a small section of the full annular junction to leave a gapped annular junction with the purpose of avoiding fluxons created inside the JTJ at the Josephson phase transition. Should any be produced, they will simply migrate through the junction extremities driven by the applied bias current needed to overcome eventual pinning potentials. This leaves the experiment only sensitive to the fluxoids produced in the ring at the phase transition.

As with our previous experiments, the present one relies on a fast heating system, obtained by integrating a Mo resistive meander line on the $4.2 \times 3 \times 0.35 \mathrm{~mm}$ Si chip containing the ring with the $\mathrm{Nb} / \mathrm{AlOx} / \mathrm{Nb}$ JTJs. The quench time $\tau_{Q}$ could be continuously varied over more that four orders of magnitude (from $20 \mathrm{~s}$ down to $1 \mathrm{~ms}$ ) by varying the width and the amplitude of the voltage pulse across the integrated resistive element. In order to determine the quench time with high accuracy, the ring temperature was monitored exploiting the well known temperature dependence of the gap voltage of high-quality $\mathrm{Nb} / \mathrm{AlOx} / \mathrm{Nb}$ JTJs already described in Ref. 10. After each ring thermal quench the critical current of the innermost JTJ is automatically stored and an algorithm has been developed for the counting of the trapped fluxoids. Finally, all the measurements have been carried out in a magnetic and electromagnetically shielded environment. During the thermal quenches all electrical connections to the heater as well as to the JTJs were disconnected. While more details on the measurement setup and on the fabrication process can be found in Refs. 13 and 14, respectively, an extensive description of the chip layout, the experimental setup and the system calibration will be given elsewhere. ${ }^{16}$

The experimental results shown in Fig. 2 were obtained using a ring with inner and outer radii, $r=30 \mu \mathrm{m}$ and $R=50 \mu \mathrm{m}$, respectively. Similar samples have shown the same behavior. We note that wider rings prevent fluxoids from tunneling out of the ring, although their smaller normal self-inductance $L_{n}$ makes fluxoid formation energetically 


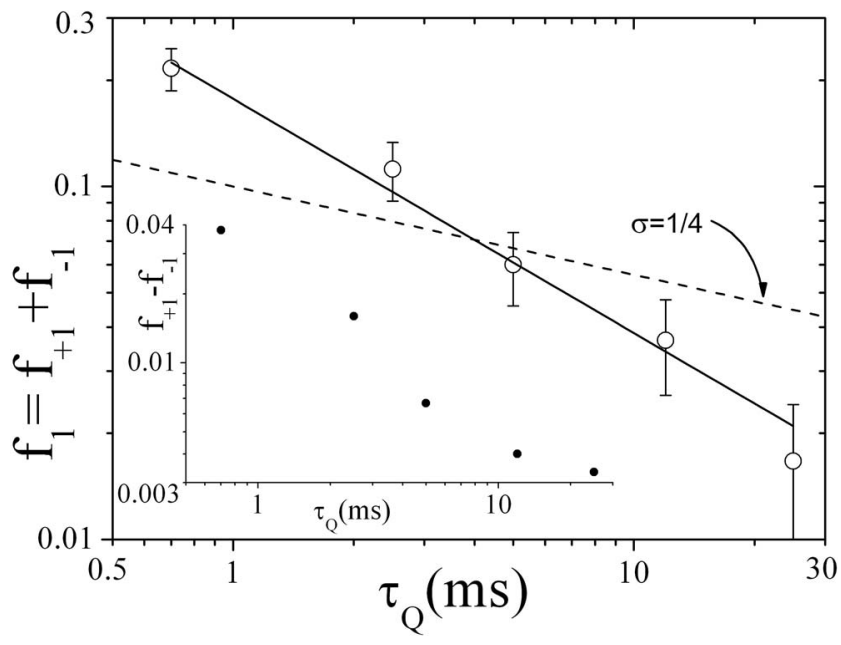

FIG. 2. Log-log plot of the measured frequency $f_{1}$ of trapping single fluxoid versus the quenching time $\tau_{Q}$ for a $\mathrm{Nb}$ ring having inner radius $r=30 \mu \mathrm{m}$, outer radius $R=50 \mu \mathrm{m}$ and thickness $d$ $=200 \mathrm{~nm} \simeq 2 \lambda_{L, N b}$. Each point corresponds to hundreds of thermal cycles. The vertical error bars gives the statistical error, while the relative error bars in $\tau_{Q}$ amounting to $\pm 10 \%$ are as large as the dots' width. The solid line is the best fit to an scaling relationship $f_{1}=a \tau_{Q}^{-b}$ which yields $a=0.18 \pm 0.02$ (taking $\tau_{Q}$ in $\mathrm{ms}$ ) and $b$ $=0.62 \pm 0.15$. For comparison purposes, the dashed line is the prediction of Eq. (9) with $\chi$ (see text) set to 0.7 to fit in ordinate scale. A similar plot for $f_{+1}-f_{-1}$ is shown in the inset.

more unlikely. In our case, the field energy $E_{0}=\Phi_{0}^{2} / 2 L_{n}$ associated with a single flux quantum $\Phi_{0}$ is several orders of magnitude larger than the thermal energy $k_{B} T_{c} / 2$ at $T_{c}{ }^{17}$

Magnetostatic numerical simulations implemented in the COMSOL multiphysics three-dimensional electromagnetics module showed that, when a single flux quantum is trapped in such rings, the radial magnetic field induced by the circulating currents at the ring inner border is as large as $1 \mathrm{~A} / \mathrm{m}$, a value easily detectable by the JTJ. For our rings, the number of trapped fluxoids was small, usually no more than one; indeed we measured the probability of trapping a single up-fluxoid (field up) $f_{+1}$ or a single down-fluxoid $f_{-1}$.

Figure 2 shows on a log-log plot the measured frequency $f_{1}=f_{+1}+f_{-1}=\left(n_{+1}+n_{-1}\right) / N=n_{1} / N$ of single fluxoid trapping, obtained by quenching the sample $N$ times for each value of a given quenching time $\tau_{O}, n_{1}$ being the number of times that one defect or one anti-defect was spontaneously produced. $N$ ranged between 250 and 300 and $n_{1}$ was never smaller than 10 , except for the rightmost point for which $n_{1}=5$. The vertical error bars gives the statistical error $f_{1} /{ } n_{1}$. The relative error bars in $\tau_{Q}$ amounting to $\pm 10 \%$ are as large as the dot's width. As expected we had $n_{+1} \approx n_{-1}$, but slightly larger than $n_{-1}$ indicating the presence of a small residual stray field in our apparatus (see inset of Fig. 2).

To test Eq. (2), we have fitted the data with a power-law function $f_{1}=a \tau_{Q}^{-b}$, with $a$ and $b$ as free fitting parameters. An instrumentally weighted least-mean-square fit of $f_{1}$ vs $\tau_{Q}$, represented by the continuous line in Fig. 2, yields $a$ $=0.18 \pm 0.02$ (taking $\tau_{Q}$ in ms) and $b=0.62 \pm 0.15$. The large fit correlation coefficient $R^{2}=0.987$ indicates that the scaling behavior is reliably confirmed, however the scaling exponent $b$ is about two times larger than expected for large loops.

A doubling of the large-loop ZK exponent for small loops has a possible explanation in the framework of the Gaussian correlation model introduced in Ref. 12 in which it was assumed that the winding number $n(x)$ is a Gaussian variable until the transition is complete, whereby all correlation functions are determined by the two-point correlation function $g\left(x_{1}-x_{2}, C\right)=\left\langle n\left(x_{1}\right) n\left(x_{2}\right)\right\rangle$. As a result ${ }^{18}$

$$
\left\langle n^{2}\right\rangle=\int_{0}^{C} \int_{0}^{C}\left\langle n\left(x_{1}\right) n\left(x_{2}\right)\right\rangle d x_{1} d x_{2}=2 C \int_{0}^{C} g(x, C) d x .
$$

For $C \ll 2 \pi \bar{\xi}$, we can assume a correlation function of the form $g(x, C)=\bar{g}(x / 2 \pi \bar{\xi}, C / 2 \pi \bar{\xi}) /(2 \pi \bar{\xi})^{2}$ so that

$$
f_{1} \approx 2 \frac{C}{2 \pi \bar{\xi}} \int_{0}^{C / 2 \pi \bar{\xi}} \bar{g}(\bar{x}, C / 2 \pi \bar{\xi}) d \bar{x} \approx 2\left(\frac{C}{2 \pi \bar{\xi}}\right)^{2} \bar{g}(0, C / 2 \pi \bar{\xi})
$$

provided $\bar{g}(\bar{x}, C / 2 \pi \bar{\xi})$ is analytic at $\bar{x}=0$. This suggests that Eq. (4) should be replaced by the scaling behavior

$$
f_{1} \approx \kappa\left(\frac{C}{2 \pi \bar{\xi}}\right)^{2}=\kappa\left(\frac{r}{\xi_{0}}\right)^{2}\left(\frac{\tau_{Q}}{\tau_{0}}\right)^{-2 \sigma},
$$

with a proportionality constant $\kappa$ of the order of unity. To buttress this suggestion, it is not difficult to show that, in the Gaussian approximation, the value of $\left\langle n^{2}\right\rangle$ along a small ring in a two-dimensional superconductor is proportional to the area enclosed by the ring. ${ }^{16}$

This doubling of the scaling exponent in Eq. (8) has the price of coming with a lower probability, but leaves us with some freedom with the ZK prefactor. Indeed, the value of the prefactor $a$ obtained from the best fit of the experimental data in Fig. 2 is about $\kappa=4-5$ times larger than the predicted value $\left(r / \xi_{0}\right)^{2} \sqrt{\tau_{0}}=0.04$ obtained using the values $r=30 \mu \mathrm{m}$, $\xi_{0} \approx 30 \mathrm{~nm}$, and $\tau_{0}=\pi \hbar / 16 k_{B} T_{c} \approx 0.16$ ps and taking $\tau_{Q}$ in $\mathrm{ms}$. As a bound we only expect agreement in the overall normalization of the prefactor $a$ to somewhat better than an order of magnitude, largely confirmed by experiment. We point out that the dependence of the prefactor $a$ on the ring width remains to be investigated both theoretically and experimentally. [We note that if Eq. (3) were true, then $\left\langle n^{2}\right\rangle$ would be $\approx 0.6$, i.e., 20 times larger than the experimental value for $\tau_{Q}=O(10 \mathrm{~ms})$, say.]

In the opposite case of large circumferences, $C \gg 2 \pi \bar{\xi}$, $g(x, C)$ is controlled by the correlation length $\bar{\xi}$ of the winding number at the time of unfreezing and does not depend on $C$, i.e., the effect of periodicity for large rings is small. With $g(x)=\bar{g}(x / 2 \pi \bar{\xi}) /(2 \pi \bar{\xi})^{2}$ on dimensional grounds, we justify the random walk assumption of Eq. (3),

$$
\left\langle n^{2}\right\rangle=\frac{C}{\pi \bar{\xi}} \int_{0}^{C / 2 \pi \bar{\xi}} \bar{g}(z) d z \approx \frac{C}{\pi \bar{\xi}} \int_{0}^{\infty} \bar{g}(z) d z=\chi \frac{C}{2 \pi \bar{\xi}},
$$

with $\chi=O(1)$. The dashed line in Fig. 2 is the prediction in Eq. (9) with $\chi$ set to 0.7 to fit to the ordinate scale.

In summary, our experiment shows reliable scaling behavior of form (3) for the creation of a single fluxoid, with 
scaling exponent $0.62 \pm 0.15$. This is obviously at variance with extrapolation (4) of Zurek prediction (3) to small rings, for which we expect $\sigma=0.25$. We have suggested that it be given by Eq. (8) with twice the exponent, as a consequence of the Gaussianity of the Cooper pair field phase (before truncation by back-reaction), an assumption supported in a slightly different context by the behavior of JTJs in an external field. ${ }^{12}$ As Gaussianity permits the instabilities from which defects form to grow as fast as possible, in general it gives the same scaling exponents as the $\mathrm{ZK}$ scenario for large systems. With this qualification we see our result as providing strong support for Zurek-Kibble scaling over a wide range of quenching time $\tau_{Q}$. We stress that this experiment confirms the Zurek-Kibble causality scenario for single isolated superconducting rings (as distinct from Josephson junctions). Further experiments to investigate the transition to the random walk regime and the effect of the ring width are planned. For example, a test of Gaussianity is that $f_{1}$ $\lesssim 0.5$ for all values of $C .^{12}$

Given that the original ZK scenario was posed to demonstrate the similarity in the role of causality at transitions in the early universe and in condensed matter systems we have seen that the finiteness of the latter systems requires careful disentangling from the underlying principles before we can draw any quantitative conclusions.

In the same vein we conclude with a speculation concerning the small-annulus JTJ experiments ${ }^{9-12}$ that, hitherto, have been the only superconductor experiments to show scaling behavior. In that case also, the observed exponent was twice that anticipated from long annuli. ${ }^{8}$ However, in the case of JTJs there is an ambiguity in their fabrication that is sufficient to double the exponent, according as the "proximity effect" enables otherwise subcritical behavior of the Josephson current density to dominate near the transition. ${ }^{10}$ We had assumed that this was the reason for the discrepancy. We shall now re-examine these earlier experiments with the above analysis in mind.

Note added in proof. It has been brought to our attention that, for BECs, numerical simulations show that there is a doubling of the ZK scaling exponent when the winding number is less than unity. See Ref. 19.

The authors thank P. Dmitriev for the sample fabrication and testing, A. Gordeeva for useful discussions, and M. Aaroe for the help at the initial stage of the experiment. *roberto@sa.infn.it

†myg@fysik.dtu.dk

${ }^{\ddagger}$ r.rivers@imperial.ac.uk

$\S$ valery@ hitech.cplire.ru

${ }^{1}$ W. H. Zurek, Nature (London) 317, 505 (1985); Acta Phys. Pol. B 24, 1301 (1993).

${ }^{2}$ W. H. Zurek, Phys. Rep. 276, 177 (1996).

${ }^{3}$ T. W. B. Kibble, Phys. Rep. 67, 183 (1980).

${ }^{4}$ J. R. Kirtley, C. C. Tsuei, and F. Tafuri, Phys. Rev. Lett. 90, 257001 (2003).

${ }^{5}$ M. Hindmarsh and A. Rajantie, Phys. Rev. Lett. 85, 4660 (2000); A. Rajantie, J. Low Temp. Phys. 124, 5 (2001).

${ }^{6}$ R. Monaco, M. Aaroe, J. Mygind, and V. P. Koshelets, J. Appl. Phys. 104, 023906 (2008).

${ }^{7}$ O. Cakir and I. O. Kulik, Phys. Rev. B 67, 174514 (2003); E. H. Brandt and J. R. Clem, ibid. 69, 184509 (2004).

${ }^{8}$ E. Kavoussanaki, R. Monaco, and R. J. Rivers, Phys. Rev. Lett. 85, 3452 (2000).

${ }^{9}$ R. Monaco, J. Mygind, and R. J. Rivers, Phys. Rev. Lett. 89, 080603 (2002).

${ }^{10}$ R. Monaco, J. Mygind, and R. J. Rivers, Phys. Rev. B 67, 104506 (2003).
${ }^{11}$ R. Monaco, J. Mygind, M. Aaroe, R. J. Rivers, and V. P. Koshelets, Phys. Rev. Lett. 96, 180604 (2006).

${ }^{12}$ R. Monaco, M. Aaroe, J. Mygind, R. J. Rivers, and V. P. Koshelets, Phys. Rev. B 77, 054509 (2008); R. Rivers, R. Monaco, J. Mygind, M. Aaroe, and V. P. Koshelets, Philos. Trans. R. Soc. London, Ser. A 366, 2871 (2008).

${ }^{13}$ R. Monaco, M. Aaroe, J. Mygind, and V. P. Koshelets, Phys. Rev. B 79, 144521 (2009).

${ }^{14}$ L. V. Filippenko, S. V. Shitov, P. N. Dmitriev, A. B. Ermakov, V. P. Koshelets, and J. R. Gao, IEEE Trans. Appl. Supercond. 11, 816 (2001).

${ }^{15}$ R. Monaco, J. Mygind, and V. P. Koshelets (unpublished).

${ }^{16}$ R. Monaco, J. Mygind, R. J. Rivers, and V. P. Koshelets (unpublished).

${ }^{17} L_{n}=\mu_{0} r[\ln (8 r / \Delta r)-1 / 2] \simeq 75 \mathrm{pH} \quad$ gives $\quad E_{0}=\Phi_{0}^{2} / 2 L_{n} \simeq 1.4$ $\times 10^{-8} \mathrm{~J}$, while, with $T_{c}=9.1 \mathrm{~K}$, the thermal energy is $k_{B} T_{c} / 2$ $=6 \times 10^{-23} \mathrm{~J}$.

${ }^{18}$ R. J. Rivers, J. Low Temp. Phys. 124, 41 (2001).

${ }^{19}$ H. Saito, Y. Kawaguchi, and M. Ueda, Phys. Rev. A 76, 043613 (2007); J. Dziarmaga, J. Meisner, and W. H. Zurek, Phys. Rev. Lett. 101, 115701 (2008). 